\title{
On the escape of cosmic rays from radio galaxy cocoons
}

\author{
T. A. Enßlin` \\ Max-Planck-Institut für Astrophysik, Karl-Schwarzschild-Str.1, 85740 Garching, Germany \\ Department of Physics, University of Toronto, 60 St. George Street, Toronto M5S1A7, Canada
}

Received 2 November 2000 / Accepted 12 November 2002

\begin{abstract}
The escape rate of cosmic ray (CR) particles from radio galaxy cocoons is a problem of high astrophysical relevance: e.g. if CR electrons are stored for long times in the dilute relativistic medium filling the radio cocoons (radio plasma in the following) they are protected against Coulomb losses and thus are able to produce a significant non-thermal Comptonisation signature on the CMB. On the other hand, CR protons and positrons which leak out of radio plasma can interact with the ambient medium, leading to characteristic gamma ray radiation by pion decay and pair annihilation. In order better understand such problems a model for the escape of CR particles from radio galaxy cocoons is presented here. It is assumed that the radio cocoon is poorly magnetically connected to the environment. An extreme case of this kind is an insulating boundary layer of magnetic fields, which can efficiently suppress particle escape. More likely, magnetic field lines are less organised and allow the transport of CR particles from the source interior to the surface region. For such a scenario two transport regimes are analysed: diffusion of particles along inter-phase magnetic flux tubes (leaving the cocoon) and cross field transport of particles in flux tubes touching the cocoon surface. The cross field diffusion is likely the dominate escape path, unless a significant fraction of the surface is magnetically connected to the environment. Major cluster merger should strongly enhance the particle escape by two complementary mechanisms. i) The merger shock waves shred radio cocoons into filamentary structures, allowing the CRs to easily reach the radio plasma boundary due to the changed morphology. ii) Also efficient particle losses can be expected for radio cocoons not compressed in shock waves. There, for a short period after the sudden injection of large scale turbulence, the (anomalous) cross field diffusion can be enhanced by several orders of magnitude. This lasts until the turbulent energy cascade has reached the microscopic scales, which determine the value of the microscopic diffusion coefficients.
\end{abstract}

Key words. ISM: cosmic rays - diffusion - magnetic fields - galaxies: intergalactic medium - galaxies: active

\section{Introduction}

\subsection{Motivation}

The outflows of radio galaxies fill large regions in the intergalactic space with relativistic, magnetised plasma. The fate of this radio plasma is unclear, since it rapidly becomes undetectable for radio telescopes due to the radiative energy losses of the higher energy electrons, which emit the observable synchrotron emission at radio bands. Lower energy electrons, and any possible present relativistic proton population, may reside for cosmological times, unless they are able to escape by spatial diffusion.

The escape of CRs out of radio plasma is of high astrophysical relevance. If CR electrons are able to leak out of the cocoon, they are possible seed particles for the Mpc-sized radio halos in clusters of galaxies if they are re-accelerated in the cluster turbulence to radio-observable energies (Giovannini et al. 1993; Brunetti et al. 2001). If radio cocoons release CR protons into the intra-cluster medium, secondary $\mathrm{CR}$ electrons are produced in hadronic interactions with the background gas,

\footnotetext{
* e-mail: ensslin@mpa-garching.mpg.de
}

which also could explain the existence of cluster radio halos (Dennison 1980). If radio plasma contains a significant fraction of positrons, they could lead to a detectable annihilation line, if they would be able to leave the radio cocoon and interact with a dense ambient intra-cluster medium (Furlanetto \& Loeb 2002).

On the other hand, if the relativistic electrons are efficiently confined for cosmological time-scales in radio cocoons, they are shielded from severe energy losses by Coulomb interaction with the environmental gas. In such a case they would be long-lived and would therefore be able to produce a nonthermal Comptonisation signature in the cosmic microwave background (Enßlin \& Kaiser 2000; Enßlin \& Sunyaev 2002). Further, if old, invisible radio plasma with confined relativistic electrons is dragged into a shock wave of the large-scale structure formation, its radio emission is possibly revived and forms the observed cluster radio relics (Enßlin \& Brüggen 2002, and references therein).

The recent detections of several ghost cavities in galaxy clusters (for lists of recent detections e.g. Enßlin \& Heinz 2002; Soker et al. 2002), which are often but not always radioemitting, support the assumption that the environmental gas 
and the bulk of the relativistic plasma stays separated on a timescale of 100 Myr. However, leakage of higher energy particles is not excluded by these observations.

CR escape from radio plasma can also play a role in our galaxy. Microquasars are proposed to contribute characteristic spectral features to the galactic CR spectrum, if CRs are able to leave the ejected plasma (Heinz \& Sunyaev 2002).

\subsection{A simplified approach}

The usual description of CR escape from systems like our Galaxy is done in terms of leaky box models, where the CR particles trapped in some region are attributed a characteristic escape time or frequency $\left(\tau=v_{\text {escape }}^{-1}\right)$. This escape time is often empirically determined (e.g. for the CR escape from the Galaxy by radioactive CR clocks (e.g. Cesarsky 1980)). It is the goal of this work to provide an estimate of this escape time and its dependency on the geometrical and plasma parameters of the system under investigation. The focus of this work is cocoons of radio galaxies, but the presented model may have other applications.

In many applications, the physical parameters will not be sufficiently constrained to allow an accurate calculation of the escape time. Nevertheless, insight into the dependencies on parameters such as turbulence, fraction of the magnetically open surface etc. allows statements of the relative CR escape rates in different situations to be made. This should help to formulate hypotheses about the conditions in which CR escape is efficient, which may be tested observationally. Examples of such tests were given in Sect. 1.1.

The following simplifications are used to compile the CR escape model:

- Convective CR transport, e.g. by plasmoids which detach by reconnection events from the source region, is neglected.

- The detailed magnetic structure of the source interior is not modelled and is treated as being homogeneous. Only the topological properties of the magnetic flux tubes touching the source surface are modelled.

- The magnetic field at the source region surface is virtually split into flux tubes with diameters of the perpendicular autocorrelation length of the magnetic field. The transport of particles along and across the flux tubes is described as a diffusion process. A similar phenomenological description of CR transport within flux tubes can be found in e.g. Chandran (2000a), where limits of such a picture are also discussed.

- It is assumed that such a surface flux tube bends at some point into the interior of the source region. This allow CRs from the interior to enter the tube there and to follow it to the surface. In the case that the magnetic fields are more onion-like in structure, the particle transport will be much more suppressed than predicted with this model. A brief discussion of such a hypothetical situation is given in Sect. 3.1.

- A simplified description of the diffusion process is adopted: the microscopic diffusion coefficients $\left(\kappa_{\|}\right.$, $\left.\kappa_{\perp}\right)$ are parametrised. The spectrum of magnetic field fluctuations, which strongly determines the transport coefficients, is assumed to be a single power law, connecting the length scales on which field line wandering happens, down to CR gyro-radii scales. In any numerical example a Kolmogorov-like turbulence spectrum is adopted. The detailed dependency on the turbulence wave types (e.g. Michalek \& Ostrowski 1997, 1998; Michalek et al. 1999; Chandran 2000b) is put into a phenomenological "fudge factor".

- The macroscopic cross field diffusion coefficient $\left(\kappa_{\mathrm{a}}\right)$ is assumed to exceed the microscopic one by orders of magnitude due to magnetic field line wandering. This allows CRs that are rapidly diffusing along the field lines to be transported across them (Rechester \& Rosenbluth 1978). This also ensures that the length scales of the diffusion problem are always shorter than length scales on which a magnetic flux tube loses its identity due to field line wandering. This should justify the use of the flux tube picture.

\subsection{The structure of the paper}

The structure of this article is the following: in Sect. 2 the theoretical tools for the description of the CR propagation in inhomogeneous media are compiled. The inter-phase CR transport is analysed in Sect. 3. Three escape routes are considered: the penetration of an isolating boundary layer (Sect. 3.1), the parallel diffusion along inter-phase magnetic flux tubes (Sect. 3.2), and the cross field escape (Sect. 3.3). In Sect. 4 the CR escape from radio plasma cocoons is investigated and in Sect. 5 the main findings of the paper are listed. In Appendix A the spatially homogenised transport equation of CRs in a smallscale inhomogeneous medium is derived. Appendix B contains a glossary of the frequently used symbols and abbreviations.

\section{Cosmic ray diffusion}

\subsection{Diffusion in inhomogeneous magnetic fields}

Since the magnetic field topology dominates the mobility of CR particles, adapted coordinates are chosen. The length $x$ is measured along the (local) mean direction $\boldsymbol{B}$ of a field bundle with local field strength $\boldsymbol{M}=\boldsymbol{B}+\delta \boldsymbol{B}$. Although individual field lines may leave the flux tubes, due to magnetic fluctuations $\delta \boldsymbol{B}$, this mean field flux tube is well defined and gives an appropriate local coordinate system.

The analysis is restricted to particles with gyro-radii much smaller than the length-scale of the magnetic fields. In this case the cross field diffusion is orders of magnitude slower than the diffusion along the field lines. Particles can be regarded as being confined in a flux tube. Since the diameter and the field strength of the tube can change as a function of $x$, it is convenient to work with the number of particles $F$ per flux tube length $\mathrm{d} x$ and magnetic flux $\mathrm{d} \phi_{B}=B \mathrm{~d} y \mathrm{~d} z$, instead of the usual volume normalisation $\mathrm{d} V=\mathrm{d} x \mathrm{~d} y \mathrm{~d} z=$ $\mathrm{d} x \mathrm{~d} \phi_{B} / B$. The particle phase space distribution function is $F(x, p, \mu, t)=\mathrm{d} N /\left(\mathrm{d} x \mathrm{~d} \phi_{B} \mathrm{~d} p \mathrm{~d} \mu\right)$, and its source density is $Q(x, p, \mu, t)=\mathrm{d} \dot{N} /\left(\mathrm{d} x \mathrm{~d} \phi_{B} \mathrm{~d} p \mathrm{~d} \mu\right) . p$ is the momentum of the particle, $v$ its velocity, $m$ is its mass, and $c$ is the speed of light. 
$\mu=p_{x} / p$ is the cosine of the pitch angle between $\boldsymbol{B}$ (or $\boldsymbol{x}$ ) and $\boldsymbol{p}$. The particle distribution is assumed to be rotationally symmetric with respect to $\boldsymbol{B}$, therefore the azimuthal angle has been integrated out. The particles entering or leaving the flux tube are included in the source term $Q$ and in the loss term $-F / \tau$ in the Fokker-Planck equation for $F$ :

$$
\begin{aligned}
\frac{\partial F}{\partial t}+\frac{\partial}{\partial x}(v \mu F)+\frac{\partial}{\partial p}(\dot{p} F)+ & \frac{\partial}{\partial \mu}(\dot{\mu} F)= \\
& \frac{\partial}{\partial \mu} D_{\mu \mu} \frac{\partial}{\partial \mu} F-\frac{F}{\tau}+Q .
\end{aligned}
$$

$D_{\mu \mu}$ is the Fokker-Planck pitch angle diffusion coefficient, which can in principle be calculated from quasi-linear theory of plasma wave-particle interaction for a given spectrum of plasma waves (e.g. Jokipii 1966, 1967; Kulsrud \& Pearce 1969; Hasselmann \& Wibberenz 1970; Skilling 1975b; Schlickeiser 2002, and references in the latter). Further Fokker-Planck diffusion coefficients, which describe momentum changes of the particles, can be found in in the literature (e.g. Schlickeiser 1989a,b). Here, momentum diffusion is ignored due its small impact on spatial transport processes, and only continuous energy or momentum losses are included by $\dot{p}$.

The continuous pitch angle changes can be calculated from the adiabatic invariants of a particle with charge $Z$ moving in spatially slowly varying, and temporally constant magnetic fields. Specifically, these are the linear momentum $p$ and the magnetic flux within a gyro-radius $\phi_{B}\left(r_{\mathrm{g}}\right)=\pi r_{\mathrm{g}}^{2} B$, where $r_{\mathrm{g}}(x, p, \mu)=\left(1-\mu^{2}\right)^{1 / 2} p c /(Z e B(x))$ is the gyro-radius. One gets

$\dot{\mu}=-\frac{v}{2}\left(1-\mu^{2}\right) \frac{\partial B}{B \partial x}$.

Klepach \& Ptuskin (1995) and Chandran (2000a) investigate the weak scattering regime, in which the mean free path of a $\mathrm{CR}$ is large compared to typical length-scales of the inhomogeneous magnetic fields and CR density scale-length. Here, a more conventional standpoint is adopted, by assuming that the particle distribution is rapidly isotropised by plasma wave interactions ${ }^{1}$. Therefore the anisotropic part of the distribution function is small compared to the isotropic one. The pitch angle integrated distribution and injection densities are defined by

$$
\begin{aligned}
& f(x, p, t)=\frac{\mathrm{d} N}{\mathrm{~d} x \mathrm{~d} \phi_{B} \mathrm{~d} p}=\int_{-1}^{1} \mathrm{~d} \mu F(x, p, \mu, t), \\
& q(x, p, t)=\frac{\mathrm{d} \dot{N}}{\mathrm{~d} x \mathrm{~d} \phi_{B} \mathrm{~d} p}=\int_{-1}^{1} \mathrm{~d} \mu Q(x, p, \mu, t) .
\end{aligned}
$$

\footnotetext{
1 This is supported by the observation that the pitch angle diffusion coefficient is the fastest of all the Fokker-Planck coefficients (e.g. Schlickeiser 2002). The relatively fast pitch angle scattering should help to maintain a nearly isotropic CR distribution - as observed in our own galaxy. A criterion to test if pitch angle scattering is indeed sufficient to establish an isotropic pitch-angle distribution - as assumed throughout this paper - is given later.
}

With the help of a quasi-linear approximation the evolution equation for $f$ can be derived from Eq. (1) following the calculation steps described in Schlickeiser (1989a):

$$
\frac{\partial f}{\partial t}+\frac{\partial}{\partial p}(\dot{p} f)=\frac{\partial}{\partial x}\left(\kappa_{\|} \frac{\partial(B f)}{B \partial x}\right)-\frac{f}{\tau}+q .
$$

This equation describes the diffusive transport of particles along the flux tube. In the case that small-scale variations of the coefficients in this equations exist, effective large-scale coefficients can be estimated, as shown in Appendix A. The diffusion coefficient is

$\kappa_{\|}(x, p)=\frac{v^{2}(p)}{8} \int_{-1}^{1} \mathrm{~d} \mu \frac{\left(1-\mu^{2}\right)^{2}}{D_{\mu \mu}(x, p, \mu)}$

and the pitch angle averaged momentum losses are

$\dot{p}(x, p)=\frac{1}{2} \int_{-1}^{1} \mathrm{~d} \mu \dot{p}(x, p, \mu)$.

In order to demonstrate that Eq. (5) is the proper transport equation the volume density of particles with momentum $p$ is introduced: $g(x, p, t)=\mathrm{d} N /(\mathrm{d} V \mathrm{~d} p)=B(x) f(x, p, t)$. Equation (5) then transforms into

$\frac{\partial g}{\partial t}+\frac{\partial}{\partial p}(\dot{p} g)=B \frac{\partial}{\partial x}\left(\frac{\kappa_{\|}}{B} \frac{\partial g}{\partial x}\right)-\frac{g}{\tau}+B q$.

This equation is consistent with Eq. (2) of Cesarsky \& Völk (1978). Diffusion of $g$ vanishes for $\partial g / \partial x=0$. This means that diffusion tries to approach a state where the space density of particles with identical momentum is constant, as it should do. Due to variations in the diameter of the flux tube this can result in a non-constant $f$ as a function of position.

\subsection{Diffusion coefficients}

The parallel diffusion coefficient depends on the pitch angle diffusion coefficient according to Eq. (6). By defining the particle-wave scattering frequency $v_{\mu}$

$v_{\mu}^{-1}=\frac{3}{8} \int_{-1}^{1} \mathrm{~d} \mu \frac{\left(1-\mu^{2}\right)^{2}}{D_{\mu \mu}(x, p, \mu)}$

one can write the parallel diffusion coefficient as

$\kappa_{\|}(x, p)=\frac{\kappa_{\mathrm{Bohm}}(x, p)}{\varepsilon(x, p)}$.

Here, $\varepsilon(x p)$ is the ratio of scattering frequency $v_{\mu}$ to the gyro-frequency $\Omega(x, p)=v(p) / r_{\mathrm{g}}(x, p, 0)$, and $\kappa_{\mathrm{Bohm}}(x, p)=$ $v(p) r_{\mathrm{g}}(x, p, 0) / 3=v(p) p c /(3 Z e B(x))$ is the Bohm diffusion coefficient. $v_{\mu}$ can be regarded as the decay time of the parallel particle velocity autocorrelations, which in the following is assumed to be identical to the decay time of the perpendicular velocity autocorrelations. This latter assumption allows us to write the microscopic perpendicular diffusion coefficient as

$\kappa_{\perp}=\frac{\varepsilon}{1+\varepsilon^{2}} \kappa_{\mathrm{Bohm}}$

(Bieber \& Matthaeus 1997). 
The scattering frequency $v_{\mu}$ depends on details of the underlying plasma turbulence on scales comparable to the gyroradius of the particle. There are two contributions important to this small-scale turbulence: the first is the Kolmogorov (or Kraichnan) cascade of large-scale turbulent kinetic energy to smaller length-scale, and the second is turbulence induced by CR streaming (Wentzel 1968, 1969; Skilling 1975a). Since the focus of this work is on the transport of poorly connected regions, the amount of CR streaming is expected to be low. In the following numerical examples, only external Kolmogorov-like turbulence is assumed. In other cases, the theory of this article can still be applied if the appropriate diffusion coefficients are used.

A simplified parameterisation of the scattering frequency is adopted here. It is assumed that

$\varepsilon(p)=\varepsilon_{0} \delta B^{2}\left(r_{\mathrm{g}}(p)\right) / B^{2}$,

where $\varepsilon_{0}$ is a fudge factor, which allows one to trace and correct the error made in this simplification, and

$\delta B^{2}(l)=\delta_{0} B^{2}\left(l / l_{B}\right)^{\gamma}$,

is the power in the magnetic fluctuations on scale $l$ (and smaller). In Kolmogorov-like turbulence $\gamma=2 / 3$, which will be used in numerical examples. The reference scale $l_{B}$ is the largest length scale which contains significant magnetic power (compared to the power-law given by Eq. (13)), so that $\delta B^{2}\left(l_{B}\right)=\left\langle\delta B^{2}\right\rangle$ is the total magnetic fluctuation power. This length scale is also of the order of the coherence length of the fields. Throughout this paper (with the exception of the enhanced anomalous diffusion discussed in Sect. 2.3) it is assumed that a single power-law describes the magnetic fluctuation spectrum from the scales important for field line wandering down to the $\mathrm{CR}$ gyro-radii.

The parameter $\delta_{0}$ and $\varepsilon_{0}$ play an important role for many of the addressed questions and their expected values should briefly be discussed here, although our knowledge of these quantities is still very limited. $\delta_{0}$ is roughly speaking the ratio of the magnetic power on the largest length-scale, on which the power-law spectrum of the inertia range of the turbulence is valid, to the total magnetic energy density. For a sharply peaked, single power-law magnetic spectrum it is $\delta_{0} \sim 1$. For a broken power-law spectrum, or a very broad maximum above the inertia-range length-scales, we expect $\delta_{0} \ll 1$. $\varepsilon_{0}$ gives the efficiency of CR scattering per magnetic power on lengthscales of the order of the particle gyro-radius, thus the power on scales which can resonate with the particle gyro-orbit. For an Alfvén wave spectrum with slab-like geometry (waves-vectors are mainly parallel to the magnetic main direction) one expects $\varepsilon_{0} \sim 1 .^{2}$ However, the nature of MHD turbulence might be anisotropic on small scales in the sense that mainly wavemodes with wave-vectors perpendicular to the main field are populated (Sridhar \& Goldreich 1994; Goldreich \& Sridhar 1997). In that case a strong reduction of the scattering frequency can be expected $\left(\varepsilon_{0} \ll 1\right)$ (a quasi-linear estimate is provided by Chandran 2000b, however it is also noted there

\footnotetext{
2 As can be found by an order of magnitude estimations of the pitch angle scattering frequencies as given by e.g. Schlickeiser (2002).
}

that the quasi-linear approximation is not fully applicable in this case).

In order that the propagation of CRs is diffusive the pitch angle distribution should be sufficiently isotropic. This is given if the scattering length $l_{\text {scatt }}(p)=v / v_{\mu}$ is small compared to any CR density scale-length $(f /(\partial f / \partial x))$. Within the above parametrisation we get

$$
\begin{aligned}
l_{\text {scatt }}(p) & =\frac{l_{B}}{\varepsilon_{0} \delta_{0}}\left(\frac{r_{\mathrm{g}}}{l_{B}}\right)^{1-\gamma} \\
& =\frac{10^{-3} \mathrm{kpc}}{\varepsilon_{0} \delta_{0}}\left(\frac{p c}{\mathrm{GeV}}\right)^{\frac{1}{3}}\left(\frac{l_{B}}{\mathrm{kpc}}\right)^{\frac{2}{3}}\left(\frac{Z B}{\mu \mathrm{G}}\right)^{-\frac{1}{3}} .
\end{aligned}
$$

This is sufficiently small in most of our cases to allow the diffusive approximation to be used.

The resulting diffusion coefficients for $\mathrm{CR}$ particles are then

$$
\begin{aligned}
\kappa_{\|} & =\frac{v l_{B}}{3 \varepsilon_{0} \delta_{0}}\left(\frac{p c}{Z e B l_{B}}\right)^{1-\gamma} \\
& =3.2 \times 10^{28} \frac{\mathrm{cm}^{2}}{\mathrm{~s}} \frac{v}{c \varepsilon_{0} \delta_{0}}\left(\frac{p c}{\mathrm{GeV}}\right)^{\frac{1}{3}}\left(\frac{l_{B}}{\mathrm{kpc}}\right)^{\frac{2}{3}}\left(\frac{Z B}{\mu \mathrm{G}}\right)^{-\frac{1}{3}} \\
\kappa_{\perp} & =\frac{v l_{B} \varepsilon_{0} \delta_{0}}{3}\left(\frac{p c}{Z e B l_{B}}\right)^{\gamma+1} \\
& =3.5 \times 10^{16} \frac{\mathrm{cm}^{2}}{\mathrm{~s}} \frac{v \varepsilon_{0} \delta_{0}}{c}\left(\frac{p c}{\mathrm{GeV}}\right)^{\frac{5}{3}}\left(\frac{l_{B}}{\mathrm{kpc}}\right)^{-\frac{2}{3}}\left(\frac{Z B}{\mu \mathrm{G}}\right)^{-\frac{5}{3}}
\end{aligned}
$$

Note that these diffusion coefficients are valid under the assumption that the turbulence dissipation length is smaller than the gyro-radius. If this is not the case, a much higher $\kappa_{\|}$and a much lower $\kappa_{\perp}$ would result.

Cross field diffusion is strongly inhibited. The small mobility of particles perpendicular to the field lines can be amplified by rapid parallel diffusion along diverging field lines (Rechester \& Rosenbluth 1978). A particle's microscopic displacement from its original field line of the order of the gyroradius by microscopic cross-field diffusion grows exponentially while it follows its new field line. This can be described in terms of a Liapunov length $\lambda_{\mathrm{L}}$, which depends on the behaviour of the magnetic field autocorrelation function at small displacements, since the old and new field lines will be strongly correlated. As soon the particle is sufficiently far from its original field line, it can be regarded as de-correlated from it. During the time needed for the particle to de-correlate from its present field line, the particle is tied to it and follows its stochastic wandering while diffusing along it. The stochastic field line wandering can be described as a diffusion process, with a diffusion coefficient $D_{B}$ which gives the perpendicular displacement (squared) per unit length travelled along the field line. Thus, during a decorrelation time the particle is doing perpendicular steps of the length given by the field line wandering times the typical diffusion length along the field line (during this time). Since this leads to a stochastic displacement, the combined propagation can be described as a macroscopic diffusion process, which is called anomalous diffusions. This anomalous diffusion allows the CRs to use the usually more powerful large-scale magnetic fluctuations for their transverse propagation. A mathematical description of this can be found in Duffy et al. (1995), which 
should be consulted for details. In their formalism the anomalous diffusion depends strongly on the parameter

$\Lambda=\frac{\delta B^{2}\left(l_{B}\right) \lambda_{\|}}{\sqrt{2} B^{2} \varepsilon\left(r_{\mathrm{g}}\right) \lambda_{\perp}}$,

where $\lambda_{\|}, \lambda_{\perp}$ are the correlation length of the field fluctuations along and perpendicular to the main field direction (usually $\left.l_{\mathrm{B}} \approx \lambda_{\|} \geq \lambda_{\perp}\right) . \Lambda$ is therefore mainly the ratio of the magnetic turbulence energy density on the largest scales $\left(l_{B}\right)$ to that on the small gyro-radius scale. For the assumed magnetic turbulence spectrum (Eq. (13)) one gets

$\Lambda=\frac{\delta_{0} \lambda_{\|}}{\sqrt{2} \varepsilon\left(r_{\mathrm{g}}\right) \lambda_{\perp}}=\frac{\lambda_{\|}}{\sqrt{2} \varepsilon_{0} \lambda_{\perp}}\left(\frac{p c}{Z e B l_{B}}\right)^{-\gamma}$.

The anomalous cross field diffusion coefficient is

$\kappa_{\mathrm{a}}=\kappa_{\perp}+\frac{2 D_{B} \kappa_{\|}}{\lambda_{\mathrm{L}} \ln \Lambda}$

for time-scales longer than the particle-field de-correlation time (Duffy et al. 1995). $D_{B}=\delta B^{2}\left(l_{B}\right) \lambda_{\|} /\left(4 B^{2}\right)$ is the field line wandering diffusion coefficient, and $\lambda_{\mathrm{L}}=B^{2} l_{\perp}^{2} /\left(\lambda_{\|} \delta B^{2}\left(l_{B}\right)\right)$ is the Liapunov length-scale ${ }^{3}$. This is the amplification length of (the expectation value of the square of) a small displacement of a particle from its initial field line while travelling along its new field line. The characteristic perpendicular length-scale $l_{\perp}$ of the size of chaotic, non-trivially twisted magnetic field fluctuations is expected by us to be of the order of $l_{B}$, although arguments exist that it might be much smaller ${ }^{4}$. With these

${ }^{3}$ Strictly speaking, only the perpendicular component of the field fluctuations $\delta B_{\perp}^{2}\left(l_{B}\right)$ enter the equations for $D_{B}$ and $\lambda_{\mathrm{L}}$. However, the difference between the perpendicular and total fluctuation levels are not very large. Since also the diffusion coefficients depend on the perpendicular magnetic fluctuations, one can interpret $\delta B^{2}$ as the perpendicular fluctuations right from the beginning and get a consistent formalism.

${ }^{4}$ Pertubatively (to lowest order in $\delta \boldsymbol{B}^{2}$ ) one can show that $l_{\perp}$ is given by the second Taylor coefficient of the magnetic field autocorrelation function perpendicular to the main field direction: $\langle\boldsymbol{\delta} \boldsymbol{B}(\boldsymbol{x}) \cdot \boldsymbol{\delta} \boldsymbol{B}(\boldsymbol{x}+$ $\left.\left.\xi_{\perp}\right)\right\rangle=\delta B^{2}\left(l_{B}\right)\left(1-\frac{1}{2} \xi_{\perp}^{2} / l_{\perp}^{2}+O\left(\xi_{\perp}^{4}\right)\right)$. More strictly speaking, $l_{\perp}$ seems to be the Taylor length of the along the mean field direction integrated and by $\lambda_{\|}$normalised autocorrelation function (of the chaotic part of the fluctuation spectrum). However, if the autocorrelation function is a direct product of a parallel and perpendicular profile, then this is identical with the simplified definition given here. Otherwise it can be expected to be close to that. However, it has been pointed out by Narayan \& Medvedev (2001) that if the chaotic (topologically non-trivially twisted) magnetic fluctuations extend from the turbulence energy injection range down to the smallest turbulence scale, the anomalous diffusion seems to become very efficient. If all the magnetic power is in such chaotic modes the effective cross field diffusion coefficient seems to be close to the order of the parallel diffusion coefficient. In the case that the magnetic turbulence in radio cocoons is completely of this chaotic nature our results would be changed in a way that particle escape from radio cocoons is always very fast. Thus the observational tests of particle escape mentioned in the introduction may confirm or refute such a picture. In the following it is assumed that the smallscale turbulence is dominated by travelling Alfvénic waves, which do not disturb the topological properties of the main field. Only on the definitions one gets

$$
\begin{aligned}
\kappa_{\mathrm{a}} & =\kappa_{\perp}\left(1+\frac{\Lambda^{2} \lambda_{\perp}^{2}}{\ln \Lambda l_{\perp}^{2}}\right) \\
& \approx \frac{v l_{B} \delta_{0} \lambda_{\|}^{2}}{6 \varepsilon_{0} \ln \Lambda l_{\perp}^{2}}\left(\frac{p c}{Z e B l_{B}}\right)^{1-\gamma} \\
& \approx 1.2 \times 10^{27} \frac{\mathrm{cm}^{2}}{\mathrm{~s}} \frac{v \delta_{0} \lambda_{\|}^{2}}{c \varepsilon_{0} l_{\perp}^{2}}\left(\frac{p c}{\mathrm{GeV}}\right)^{\frac{1}{3}}\left(\frac{l_{B}}{\mathrm{kpc}}\right)^{\frac{2}{3}}\left(\frac{Z B}{\mu \mathrm{G}}\right)^{-\frac{1}{3}}
\end{aligned}
$$

The ratio of the anomalous to parallel diffusion coefficients

$\kappa_{\mathrm{a}} / \kappa_{\|}=\frac{\delta_{0}^{2} \lambda_{\|}^{2}}{2 \ln \Lambda l_{\perp}^{2}} \approx 0.037 \delta_{0}^{2} \lambda_{\|}^{2} / l_{\perp}^{2}$

is nearly independent of the particle momentum, and it is independent of the fudge factor $\varepsilon_{0}$. The numerical result is in good agreement with detailed Monte-Carlo simulation of particle diffusion in slab-geometry and homogeneous turbulence by Giacalone \& Jokipii (1999). These authors find a ratio of $\kappa_{\mathrm{a}} / \kappa_{\|}=0.02 \ldots 0.04$ for a turbulence strength comparable with $\delta_{0}=1$ (see their Fig. 3) and a scaling which seems to be only slightly weaker than $\propto \delta_{0}^{2}$ (see their Fig. 4).

\subsection{Enhanced anomalous diffusion}

Under certain circumstances the anomalous cross field diffusion is extremely efficient. The anomalous diffusion coefficient $\kappa_{\mathrm{a}}$ depends quadratically on $\Lambda$ (Eq. (20)), which is mainly the ratio of large-scale to small scale turbulence (Eq. (18)). $\Lambda$ is therefore independent of the level of the turbulence, as long as the spectral shape of the turbulence energy spectrum is not changed. Increasing the turbulence energy density of a system by a factor $X_{\mathrm{T}}$ increases $\kappa_{\mathrm{a}}$ also by $X_{\mathrm{T}}$, due to the dependence of $\kappa_{\perp} \propto X_{\mathrm{T}}\left(\right.$ or $\left.\kappa_{\|} \propto X_{\mathrm{T}}^{-1}\right)$ on the small scale turbulence.

If the slope of the turbulence is changed, drastic changes in $\kappa_{\mathrm{a}}$ can result. If e.g. large-scale kinetic energy is suddenly injected into a system, it may need a period on the order of the eddy turnover time $\tau_{\mathrm{T}}=l_{\mathrm{T}} / v_{\mathrm{T}}$ before the turbulent cascade has also raised the small-scale turbulence level. During this period the microscopic diffusion coefficients $\kappa_{\|}$and $\kappa_{\perp}$ stay unchanged, but $\kappa_{\mathrm{a}}$ is increased by a factor of $X_{\mathrm{T}}^{2}$.

\section{Cosmic ray escape}

\subsection{Boundary layer penetration}

If a boundary layer of insulating magnetic fields (without any flux leaving the boundary layer regions) separates the interior from the surrounding of the source region the escape of cosmic ray particles is strongly inhibited. Although radio polarization observations of radio cocoons indicate that magnetic fields are well aligned with the cocoon surface (e.g. Laing 1980, 1981; Spangler et al. 1984), it is far from obvious if this implies an insulating boundary layer. The origin of such a hypothetical

largest scales we do expect strong non-trivial topologies, produced by the turbulence driving forces. Their strength should be limited (and therefore possibly confined from the smaller-scales) by reconnection events. Therefore we expect $l_{\perp} \sim l_{B}$. 
layer may be due to mixing of radio plasma with the ambient gas in a thin surface mixing layer, which is then dynamically decoupled from the turbulent interior due to its much higher inertia. The expected shear between the interior and the mixing layer may amplify and align magnetic fields into such an insulating boundary layer.

As a simplified model we assume the source region to be spherical with radius $r_{\mathrm{s}}$, the boundary layer to have a thickness $d_{\mathrm{b}}$. The layer, which extends from radius $r_{\mathrm{i}}=r_{\mathrm{s}}-d_{\mathrm{b}}$ to $r_{\mathrm{s}}$, is assumed to be filled with magnetic fields which are mainly tangential to the source surface at $r_{\mathrm{s}}$. Therefore the radial diffusion coefficient is that of the macroscopic cross field diffusion $\kappa_{\mathrm{a}}$. In a quasi-stationary situation (slow particle escape) the space density of particles is given by

$g(r, p)=g_{0}(p) \frac{r^{-1}-r_{\mathrm{s}}^{-1}}{r_{\mathrm{i}}^{-1}-r_{\mathrm{s}}^{-1}}$

within the boundary layer $\left(r_{\mathrm{i}}<r<r_{\mathrm{s}}\right)$, where $g_{0}(p)$ is the particle spectrum inside the layer $\left(r<r_{\mathrm{i}}\right)$. From this the number of CRs within the source region is given by

$N(p)=\int \mathrm{d} V_{\mathrm{s}} g(r, p)=\frac{2 \pi}{3} g_{0}(p) r_{\mathrm{s}} r_{\mathrm{i}}\left(r_{\mathrm{s}}+r_{\mathrm{i}}\right)$,

and the $\mathrm{CR}$ escape rate through the source surface area $A_{\mathrm{s}}$ is given by

$\dot{N}(p)=\left.A_{\mathrm{s}} \kappa_{\mathrm{a}} \nabla g(r, p)\right|_{r=r_{\mathrm{s}}}=-\frac{4 \pi \kappa_{\mathrm{a}} g_{0}(p)}{r_{\mathrm{i}}^{-1}-r_{\mathrm{s}}^{-1}}$.

The escape frequency follows to be

$$
\begin{aligned}
v_{\text {layer }}(p) & =-\frac{\dot{N}(p)}{N(p)}=\frac{6 \kappa_{\mathrm{a}}(p)}{r_{\mathrm{s}}^{2}-r_{\mathrm{i}}^{2}}=\frac{3 \kappa_{\mathrm{a}}(p)}{r_{\mathrm{s}} d_{\mathrm{b}}\left(1-d_{\mathrm{b}} /\left(2 r_{\mathrm{s}}\right)\right)} \\
& \approx \frac{4.0}{\mathrm{Gyr}} \frac{v \delta_{0}}{c \varepsilon_{0}} \frac{\lambda_{\|}^{2} l_{B}^{\frac{2}{3}} \mathrm{kpc}^{\frac{4}{3}}}{r_{\mathrm{s}} d_{\mathrm{b}} l_{\perp}^{2}}\left(\frac{p c}{\mathrm{GeV}}\right)^{\frac{1}{3}}\left(\frac{Z B}{\mu \mathrm{G}}\right)^{-\frac{1}{3}} .
\end{aligned}
$$

It should be noted that enhanced anomalous diffusion due to a sudden raise of the large-scale turbulence level by a factor $X_{\mathrm{T}}$ increases the particle escape rate through a boundary layer by a factor $X_{\mathrm{T}}^{2}$ during the period of not fully developed turbulence, and $X_{\mathrm{T}}$ afterwards (see Sect. 2.3).

\subsection{Flux tube escape route}

If some magnetic flux $\phi_{B}$ leaks from the source to the loss region, the CR can escape following the field lines. A possible situation is sketched in Fig. 1. The leakage requires that the particles of the source region enter the inter-phase flux tube either by perpendicular diffusion from disconnected regions, or by travelling along the flux tube from a distant reservoir. Then they diffuse through a transition zone between the two phases and finally leave the tube in the loss region by cross field diffusion (or escape to infinity along the field line).

For simplicity it is assumed that within the source and the loss regions all parameters of Eq. (5) are spatially constant. The source function $q$ is given by $f_{\mathrm{s}}$, which is the average density of particles within the source (per magnetic flux) divided by the time $\tau_{\mathrm{s}}$ a particle needs to cross-field diffuse over the distance

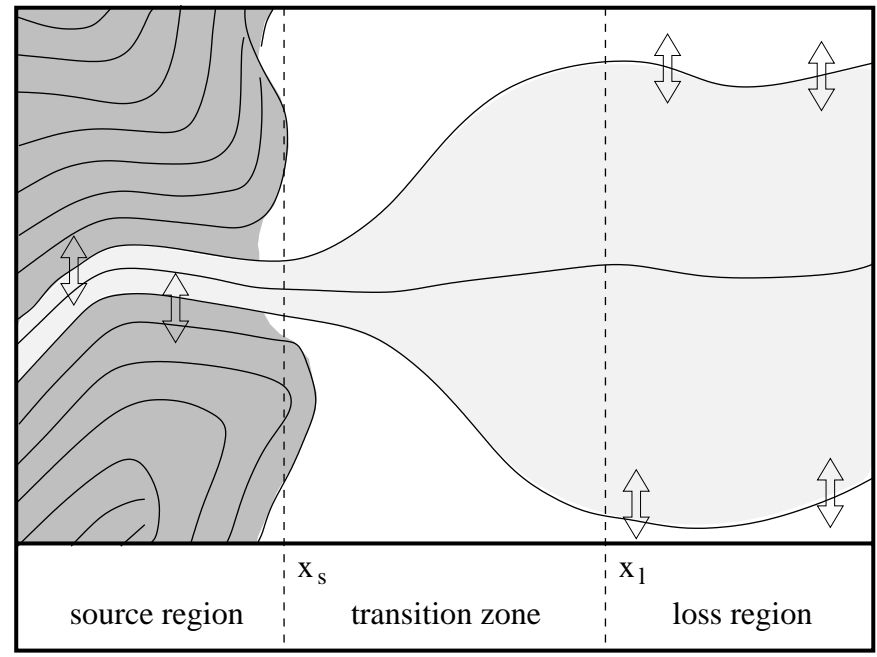

Fig. 1. Geometry of the inter-phase region. The left $\left(x<x_{\mathrm{s}}\right)$ and right regions $\left(x_{1}<x\right)$ are the source and loss regions of the particle. A bundle of field lines connect these regions through a transition zone $\left(x_{\mathrm{s}}<x<x_{1}\right)$.

given by the diameter of the flux tube. The typical time a particle requires to enter or leave a flux tube with flux $\phi=\pi r^{2} B$ and radius $r \approx \lambda_{\perp} / 2$ is given by $\tau=r^{2} /\left(4 \kappa_{\mathrm{a}}\right)=\phi /\left(4 \pi B \kappa_{\mathrm{a}}\right)$. In the loss region $q=0$. In the transition zone $\kappa_{\|}$and $B$ might be functions of the position, but no particle losses or sources are assumed there. Furthermore, any energy loss processes are ignored. These conditions lead to the following parameters:

$$
\begin{aligned}
q(x, p)= & H\left(x_{\mathrm{s}}-x\right) f_{\mathrm{s}}(p) / \tau_{\mathrm{s}}(p), \\
\tau(x, p)= & H\left(x_{\mathrm{s}}-x\right) \tau_{\mathrm{s}}(p)+H\left(x-x_{1}\right) \tau_{1}(p), \\
\dot{p}(x, p)= & 0, \\
B(x)= & H\left(x_{\mathrm{s}}-x\right) B_{\mathrm{s}}+H\left(x-x_{1}\right) B_{1} \\
& +H\left(x-x_{\mathrm{s}}\right) H\left(x_{1}-x\right) B_{\mathrm{t}}(x), \text { and, } \\
\kappa_{\|}(x, p)= & H\left(x_{\mathrm{s}}-x\right) \kappa_{\mathrm{s}}(p)+H\left(x-x_{1}\right) \kappa_{\mathrm{l}}(p), \\
& +H\left(x-x_{\mathrm{s}}\right) H\left(x_{1}-x\right) \kappa_{\mathrm{t}}(x, p) .
\end{aligned}
$$

$H(x)$ is the Heaviside step function. Since the momentum is conserved $(\dot{p}(x, p)=0)$, Eq. (5) can be solved independently for all momenta. The explicit dependency on $p$ is dropped in the following. The solution of Eq. (5) with the parameters given above, smooth field lines $\left(\mathrm{d} B / \mathrm{d} x=0\right.$ at $x_{\mathrm{s}}$ and $\left.x_{1}\right)$ and the boundary conditions $f(-\infty)=f_{\mathrm{s}}, f(\infty)=0$ is

$f(x)= \begin{cases}f_{\mathrm{s}}-\left(f_{\mathrm{s}}-f_{\mathrm{a}}\right) \exp \left(\left(x-x_{\mathrm{s}}\right) / l_{\mathrm{s}}\right) & ; x<x_{\mathrm{s}} \\ {\left[B_{\mathrm{s}} f_{\mathrm{a}}-\left(B_{\mathrm{s}} f_{\mathrm{a}}-B_{1} f_{\mathrm{b}}\right) \sigma(x)\right] / B_{\mathrm{t}}(x)} & ; x_{\mathrm{s}}<x<x_{1}, \\ f_{\mathrm{b}} \exp \left(\left(x_{\mathrm{l}}-x\right) / l_{1}\right) & ; x_{\mathrm{l}}<x\end{cases}$

with

$l_{\mathrm{s}}=\sqrt{\kappa_{\mathrm{s}} \tau_{\mathrm{s}}}, \quad l_{1}=\sqrt{\kappa_{\mathrm{l}} \tau_{1}}$,

$f_{\mathrm{a}}=f_{\mathrm{s}} /\left[1+1 /\left(\frac{\kappa_{\mathrm{s}} I}{l_{\mathrm{s}} B_{\mathrm{s}}}+\frac{\kappa_{\mathrm{s}} l_{\mathrm{l}} B_{1}}{\kappa_{\mathrm{l}} l_{\mathrm{s}} B_{\mathrm{s}}}\right)\right]$,

$f_{\mathrm{b}}=f_{\mathrm{s}} /\left[\frac{\kappa_{\mathrm{l}} I}{l_{1} B_{\mathrm{s}}}+\frac{B_{1}}{B_{\mathrm{s}}}+\frac{\kappa_{1} l_{\mathrm{s}}}{\kappa_{\mathrm{s}} l_{1}}\right]$,

$\sigma(x)=\frac{1}{I} \int_{x_{\mathrm{s}}}^{x} \mathrm{~d} x^{\prime} \frac{B_{\mathrm{t}}\left(x^{\prime}\right)}{\kappa_{\mathrm{t}}\left(x^{\prime}\right)}$, and $I=\int_{x_{\mathrm{s}}}^{x_{1}} \mathrm{~d} x^{\prime} \frac{B_{\mathrm{t}}\left(x^{\prime}\right)}{\kappa_{\mathrm{t}}\left(x^{\prime}\right)}$. 


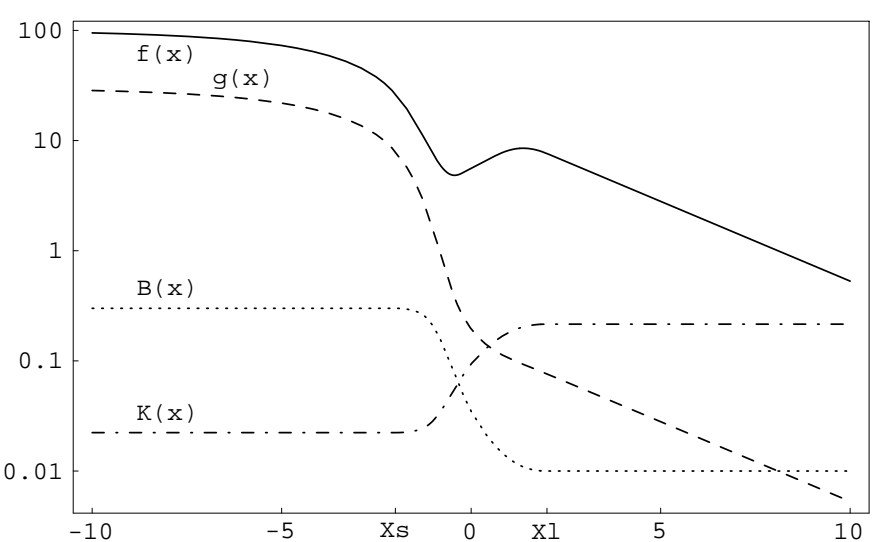

Fig. 2. Profiles of $f(x), g(x)=B(x) f(x), B(x)$, and $\kappa_{\|}(x)$. The parameters are $f_{\mathrm{s}}=100, B_{\mathrm{s}}=0.3, B_{1}=0.01, x_{\mathrm{s}}=-2, x_{1}=2$, $l_{\mathrm{s}}=3, l_{1}=3$. The field in the transition zone is parametrised by $B_{\mathrm{t}}(x)=\left[B_{1}-\left(B_{1}-B_{\mathrm{s}}\right) \exp \left(-\sqrt{B_{\mathrm{s}} / B_{1}}\left(x-x_{\mathrm{s}}\right)^{2} /\left(x_{1}-x\right)^{2}\right)\right]+B_{\mathrm{s}}-\left(B_{\mathrm{s}}-\right.$ $\left.B_{1}\right) \exp \left(-\sqrt{B_{1} / B_{\mathrm{s}}}\left(x-x_{1}\right)^{2} /\left(x_{\mathrm{s}}-x\right)^{2}\right) / 2$. Further $\kappa_{\|}(x)=0.01 B^{-2 / 3}(x)$ is assumed.

An example density profile is shown in Fig. 2 for strong fields in the source and weak field in the loss region, and in Fig. 3 for the reversed geometry.

If one introduces the spatial average within the transition zone by $\langle A(x)\rangle=\int_{x_{\mathrm{s}}}^{x_{1}} \mathrm{~d} x A(x) / l_{\mathrm{t}}$, where $l_{\mathrm{t}}=x_{1}-x_{\mathrm{s}}$, one can write

$I=\bar{B}_{\mathrm{t}} l_{\mathrm{t}} / \bar{\kappa}_{\mathrm{t}}=\bar{B}_{\mathrm{t}} \sqrt{\tau_{\mathrm{t}} / \bar{\kappa}_{\mathrm{t}}}$, where

$\bar{B}_{\mathrm{t}}=\langle B\rangle, \bar{\kappa}_{\mathrm{t}}=\bar{B}_{\mathrm{t}} /\left\langle B / \kappa_{\mathrm{t}}\right\rangle$, and $\tau_{\mathrm{t}}=l_{\mathrm{t}}^{2} / \bar{\kappa}_{\mathrm{t}}$.

If $B$ changes by a large factor in the transition zone one expects $\max \left(B_{\mathrm{s}}, B_{1}\right)>\bar{B}_{\mathrm{t}} \gg \min \left(B_{\mathrm{s}}, B_{1}\right)$. Due to the expected anticorrelation of $B$ and $\kappa_{\|}$also $\max \left(\kappa_{\mathrm{s}}, \kappa_{\mathrm{l}}\right) \gg \bar{\kappa}_{\mathrm{t}}>\min \left(\kappa_{\mathrm{s}}, \kappa_{\mathrm{l}}\right)$ is likely.

The total particle loss of the source into the loss region is given by

$-\dot{N}_{\mathrm{s} \|}=\int_{x_{1}}^{\infty} \mathrm{d} x \frac{f(x)}{\tau_{1}}=\frac{f_{\mathrm{s}} \phi_{B} B_{\mathrm{s}}}{B_{\mathrm{s}} \sqrt{\tau_{\mathrm{s}} / \kappa_{\mathrm{s}}}+\bar{B}_{\mathrm{t}} \sqrt{\tau_{\mathrm{t}} / \bar{\kappa}_{\mathrm{t}}}+B_{1} \sqrt{\tau_{1} / \kappa_{1}}}$.

If several loss channels exist with similar properties $\left(\tau_{\mathrm{s}}, \tau_{1}\right)$ then $\phi_{B}$ in Eq. (39) can be replaced by the sum of the absolute fluxes of these channels. In this case the flux leaving the source region is $\phi_{\mathrm{B}}=A_{\mathrm{s}} \eta_{\mathrm{s}} B_{\mathrm{s}}$, where $\eta_{\mathrm{s}}$ is the fraction of the source surface $\left(A_{\mathrm{s}}\right)$ penetrated by inter-phase magnetic flux. After defining the characteristic length-scale of the source by $L_{\mathrm{s}}=V_{\mathrm{s}} / A_{\mathrm{s}}$, where $V_{\mathrm{s}}$ is the source volume, and noticing that $N_{\mathrm{s}}=V_{\mathrm{s}} g_{\mathrm{s}}=V_{\mathrm{s}} f_{\mathrm{s}} B_{\mathrm{s}}$ one can write the typical particle escape frequency $v_{\|}$as

$v_{\|}=-\frac{\dot{N}_{\mathrm{s} \|}}{N_{\mathrm{s}}}=\frac{\eta_{\mathrm{s}} B_{\mathrm{s}} / L_{\mathrm{s}}}{B_{\mathrm{s}} \sqrt{\tau_{\mathrm{s}} / \kappa_{\mathrm{s}}}+\bar{B}_{\mathrm{t}} \sqrt{\tau_{\mathrm{t}} / \bar{\kappa}_{\mathrm{t}}}+B_{1} \sqrt{\tau_{1} / \kappa_{1}}} \cdot$

This formula is simplified if one of the terms in the denominator dominates. Two cases correspond to typical astrophysical situations:

Strongly magnetised source region:

$v_{\|} \approx \frac{\eta_{\mathrm{s}}}{L_{\mathrm{s}}} \sqrt{\frac{\kappa_{\mathrm{s}}}{\tau_{\mathrm{s}}}}=\frac{\eta_{\mathrm{s}} \kappa_{\mathrm{s}}}{L_{\mathrm{s}} l_{\mathrm{s}}}=\frac{\phi_{B} \kappa_{\mathrm{s}}}{B_{\mathrm{s}} V_{\mathrm{s}} l_{\mathrm{s}}}$.

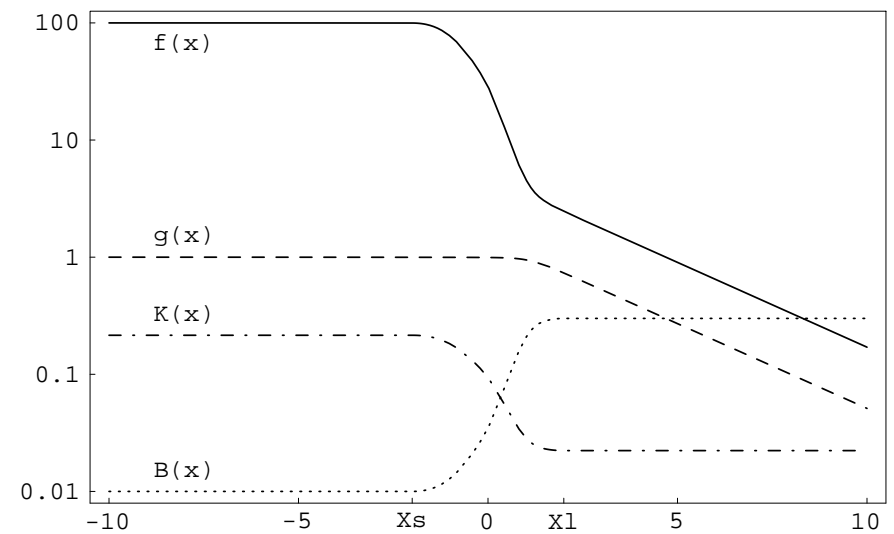

Fig. 3. As Fig. 2, but $B_{\mathrm{s}}=0.01, B_{1}=0.3$.

Strongly magnetised loss region:

$v_{\|} \approx \frac{\eta_{\mathrm{s}} B_{\mathrm{s}}}{L_{\mathrm{s}} B_{1}} \sqrt{\frac{\kappa_{\mathrm{l}}}{\tau_{1}}}=\frac{\eta_{\mathrm{s}} B_{\mathrm{s}} \kappa_{1}}{L_{\mathrm{s}} l_{1} B_{1}}=\frac{\phi_{B} \kappa_{1}}{B_{1} V_{\mathrm{s}} l_{1}}$

If two regions 1 and 2 with volumes $V_{1}$ and $V_{2}$ are connected by some magnetic flux $\phi_{\mathrm{B}}$, then the ratio of the particle exchange frequencies is equal to the inverse volume ratio, as it is required by detailed balance:

$\frac{v_{1 \rightarrow 2}}{v_{2 \rightarrow 1}}=\frac{\eta_{1} B_{1} / L_{1}}{\eta_{2} B_{2} / L_{2}}=\frac{V_{2}}{V_{1}}$

In the following it is assumed that the source field region has the strongest magnetic fields and therefore Eq. (41) is used. If a number of $M_{\|}$flux-tubes with diameter $\lambda_{\perp}$ each leave the source region the total magnetic flux leaving the source is $\phi_{B}=$ $M_{\|} \pi B \lambda_{\perp}^{2} / 4$. Applying the identity $M_{\|}=4 \eta_{\mathrm{s}} A_{\mathrm{s}} /\left(\pi \lambda_{\perp}^{2}\right)$ one finds that the particle loss frequency is then given by

$$
\begin{aligned}
v_{\|} & \approx \frac{4 \eta_{\mathrm{s}} \sqrt{\kappa_{\mathrm{a}} \kappa_{\|}}}{\lambda_{\perp} L_{\mathrm{s}}} \\
& =\frac{4 \eta_{\mathrm{s}} l_{\mathrm{B}} \lambda_{\|} v}{3 \sqrt{2 \ln \Lambda} \varepsilon_{0} l_{\perp} \lambda_{\perp} L_{\mathrm{s}}}\left(\frac{p c}{Z e B l_{B}}\right)^{1-\gamma} \\
& \approx \frac{0.081}{\mathrm{Myr}} \eta_{\mathrm{s}} \frac{v}{c \varepsilon_{0}} \frac{l_{B}^{2 / 3} \lambda_{\|} /\left(\lambda_{\perp} l_{\perp} L_{\mathrm{s}}\right)}{\mathrm{kpc}^{-4 / 3}}\left(\frac{p c /(Z B)}{\mathrm{GeV} / \mu \mathrm{G}}\right)^{\frac{1}{3}} .
\end{aligned}
$$

It should be noted that enhanced anomalous diffusion due to a sudden raise of the large-scale turbulence level by a factor $X_{\mathrm{T}}$ increases the flux tube particle escape rate by a factor $X_{\mathrm{T}}$ only during the period of not fully developed turbulence, but not afterwards (see Sect. 2.3).

If the CRs escape rapidly from the source region, they can excite plasma waves which again scatter the CRs. This limits the escape velocity to approximately the Alfvén- or thermal velocity, or whichever is larger (Tademaru 1969; Holman et al. 1979). For the high energy part of the CR spectrum the limiting velocity can be even larger, since the small number density of high energy CRs in typical power-law distributions does not lead to an efficient excitation of the scattering waves 
(e.g. Felice \& Kulsrud 2001). However, an important quantity is the CR escape velocity at the surface of the source region:

$$
\begin{aligned}
v_{\mathrm{CR}} & =\frac{\kappa_{\mathrm{s}}}{l_{\mathrm{s}}}=\frac{4 v}{3 \varepsilon_{0} \sqrt{2 \ln \Lambda}} \frac{l_{B} \lambda_{\|}}{\lambda_{\perp} l_{\perp}}\left(\frac{p c}{Z e B l_{B}}\right)^{1-\gamma} \\
& \approx 80 \frac{\mathrm{km}}{\mathrm{s}} \frac{v}{\varepsilon_{0} c} \frac{l_{B}^{5 / 3} \lambda_{\|}}{\lambda_{\perp} l_{\perp} \mathrm{kpc}^{2 / 3}}\left(\frac{p c /(Z B)}{\mathrm{GeV} / \mu \mathrm{G}}\right)^{\frac{1}{3}} .
\end{aligned}
$$

As long as this velocity is below the limiting speed, which for radio galaxy cocoons should be the environmental sound speed, CR self-confinement due to wave excitation should be negligible.

\subsection{Cross field escape route}

In order to model the cross field escape frequency an individual flux tube of diameter $\lambda_{\perp}$ is investigate first, which touches the inter phase surface on a length-scale $\lambda_{\|}$(see Fig. 4). The particle distribution function along this tube is governed by Eq. (5) if one adopts the following parameters:

$q(x, p)=\left(1-\sigma_{\perp}(x)\right) f_{\mathrm{s}}(p) / \tau_{\mathrm{s}}(p)$,

$\tau(x, p)=\tau_{\mathrm{s}}(p)$,

$\dot{p}(x, p)=0$,

$B(x)=B_{\mathrm{S}}$

$\kappa_{\|}(x, p)=\kappa_{\mathrm{S}}(p)$.

$\sigma_{\perp}(x)$ is the fraction of the flux tubes surface which is in contact with the other phase. $\sigma_{\perp}(x)=0$ for $x<x_{1}$ or $x>x_{2}$. Again, the notation of the momentum dependence is dropped for convenience. The solution can be found with the help of the method of Green's functions and is given by

$f(x)=f_{\mathrm{s}}\left[1-\frac{1}{2 l_{\mathrm{s}}} \int_{x_{1}}^{x_{2}} \mathrm{~d} x^{\prime} \sigma_{\perp}\left(x^{\prime}\right) \exp \left(-\left|x-x^{\prime}\right| / l_{\mathrm{s}}\right)\right]$.

In the following it is assumed that $\sigma_{\perp}(x)=$ const within the length $\lambda_{\|}=x_{2}-x_{1}$. In this case

$f(x)=f_{\mathrm{s}}\left[1-\frac{\sigma_{\perp}}{2}\left(h\left(\frac{x-x_{1}}{l_{\mathrm{s}}}\right)+h\left(\frac{x_{2}-x}{l_{\mathrm{s}}}\right)\right)\right]$, with

$h(z)=\operatorname{sgn}(z)\left(1-e^{-|z|}\right)$,

which is shown in Fig. 5. The particle escape rate (into the other phase) of this filament is then

$-\dot{N}_{\perp}=\frac{f_{\mathrm{s}} \lambda_{\|} \sigma_{\perp}}{\tau_{\mathrm{s}}}\left[1-\frac{\sigma_{\perp}}{y}\left(y+e^{-y}-1\right)\right]$,

where

$y=\frac{\lambda_{\|}}{l_{\mathrm{s}}}=\frac{\lambda_{\|}}{\sqrt{\kappa_{\mathrm{s}} \tau_{\mathrm{s}}}}=\frac{\lambda_{\|}^{2}}{l_{\perp} \lambda_{\perp}} \frac{4 \delta_{0}}{\sqrt{2 \ln \Lambda}}$.

Usually $y \ll 1$, otherwise the situation resembles more the isolating boundary layer case discussed in Sect. 3.1. The number $M_{\perp}$ of flux tubes which touch that part of the surface of the source region, which is not intersected by inter phase magnetic flux, is

$M_{\perp}=\frac{A_{\mathrm{s}}\left(1-\eta_{\mathrm{s}}\right)}{\pi \lambda_{\perp} \lambda_{\|} \sigma_{\perp}}$

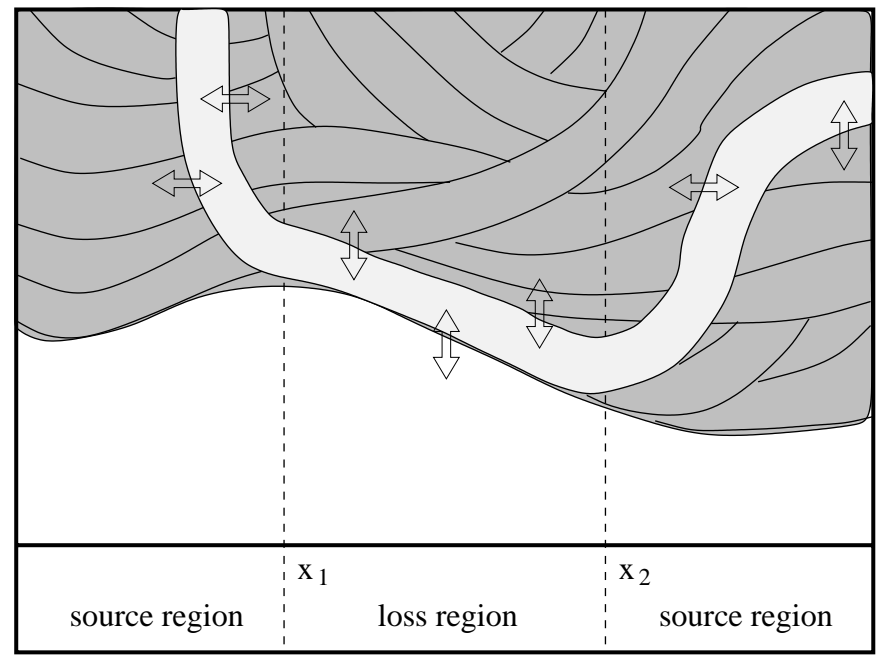

Fig. 4. Geometry of a flux tube which is part of the source boundary. The left $\left(x<x_{1}\right)$ and right regions $\left(x_{2}<x\right)$ are the source and loss regions of the particles. In between, escape into the other phase is possible.

This leads to perpendicular escape frequency of

$v_{\perp}=-\frac{M_{\perp} \dot{N}_{\perp}}{N_{\mathrm{s}}}=v_{\perp, 0}\left(1-\eta_{\mathrm{s}}\right)\left[1-\frac{\sigma_{\perp}}{y}\left(y+e^{-y}-1\right)\right]$,

with

$$
\begin{aligned}
v_{\perp, 0} & =\frac{A_{\mathrm{s}} \lambda_{\perp}}{4 V_{\mathrm{s}} \tau_{\mathrm{s}}}=\frac{4 \kappa_{\mathrm{a}}}{L_{\mathrm{s}} \lambda_{\perp}} \\
& =\frac{2 \delta_{0}}{3 \varepsilon_{0} \ln \Lambda} \frac{l_{B} \lambda_{\|}^{2} v}{l_{\perp}^{2} \lambda_{\perp} L_{\mathrm{s}}}\left(\frac{p c}{Z e B l_{B}}\right)^{\frac{1}{3}} \\
& \approx \frac{0.016}{\mathrm{Myr}} \frac{\delta_{0} v}{\varepsilon_{0} c} \frac{l_{B}^{2 / 3} \lambda_{\|}^{2} /\left(l_{\perp}^{2} \lambda_{\perp} L_{\mathrm{s}}\right)}{\mathrm{kpc}^{-4 / 3}}\left(\frac{p c /(Z B)}{\mathrm{GeV} / \mu \mathrm{G}}\right)^{\frac{1}{3}} .
\end{aligned}
$$

The reverse process, in which particles enter the strongly magnetised region, has a frequency which can be calculated from the escape frequency (Eq. (56)) and the condition of detailed balance (Eq. (43)).

It should be noted that enhanced anomalous diffusion due to a sudden raise of the large-scale turbulence level by a factor $X_{\mathrm{T}}$ increases the cross field particle escape rate by a factor $X_{\mathrm{T}}^{2}$ during the period of not fully developed turbulence, and $X_{\mathrm{T}}$ afterwards (see Sect. 2.3).

\subsection{The best escape route}

The most efficient escape route can be found by comparing the escape frequencies for the flux tube and the cross field escape route:

$$
\begin{aligned}
\frac{v_{\perp}}{v_{\|}} & =\sqrt{\frac{\kappa_{\mathrm{a}}}{\kappa_{\|}}} \frac{1-\eta_{\mathrm{s}}}{\eta_{\mathrm{s}}}\left[1-\frac{\sigma_{\perp}}{y}\left(y+e^{-y}-1\right)\right] \\
& \approx \frac{\delta_{0} \lambda_{\|}}{5 l_{\perp} \eta_{\mathrm{s}}} .
\end{aligned}
$$

During the injection phase of turbulence (a few $\tau_{\mathrm{T}}$ ) the ratio $v_{\perp} / v_{\|}$is increased by an additional factor of $X_{\mathrm{T}}$ over the estimates given in Eq. (60). Under certain conditions 


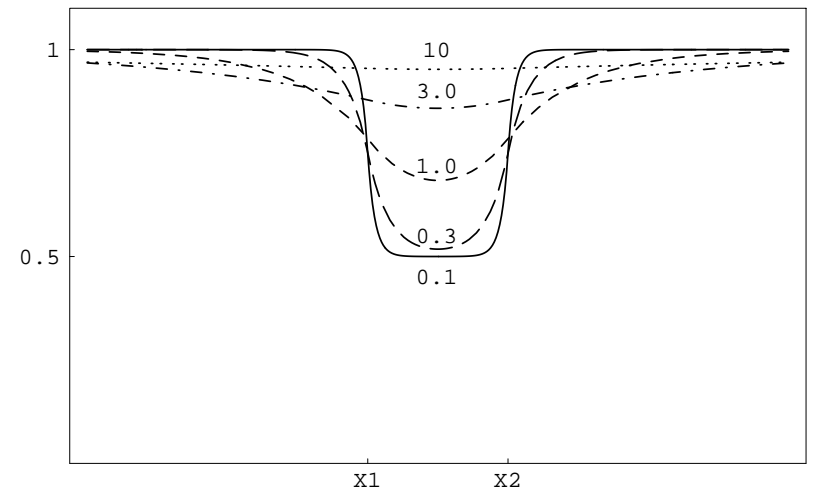

Fig. 5. Profile of $f(x)$ for $\sigma_{\perp}=0.5, \lambda_{\|}=2$ and different $l_{\mathrm{s}}$ as labelled in the graph.

$\left(\delta_{0}<5 \eta_{\mathrm{s}}<X_{\mathrm{T}} \delta_{0}\right)$ the dominant escape mechanism can switch temporarily from flux tube escape to cross field escape during the onset of turbulence.

\section{CR escape from radio plasma}

\subsection{A quiet environment}

The old radio plasma cocoon of a small radio galaxy in the central IGM of a galaxy cluster might be of approximately spherical shape. Here the following parameters are assumed: $B=10 \mu \mathrm{G}, l_{B}=\lambda_{\|}=3 \mathrm{kpc}, l_{\perp}=\lambda_{\perp}=1 \mathrm{kpc}$, and a cocoon diameter of $50 \mathrm{kpc}$. The turbulence inside old radio plasma is likely the turbulence induced by the environment, and therefore assumed to be Kolmogorov-like. The diffusion coefficients of $10 \mathrm{GeV} \mathrm{CR}$ particles are then $\kappa_{\|}=7 \times 10^{28} \mathrm{~cm}^{2} \mathrm{~s}^{-1} /\left(\varepsilon_{0} \delta_{0}\right)$, $\kappa_{\perp}=1.7 \times 10^{16} \mathrm{~cm}^{2} \mathrm{~s}^{-1} \varepsilon_{0} \delta_{0}$, and $\kappa_{\mathrm{a}}=1.9 \times 10^{28} \mathrm{~cm}^{2} \mathrm{~s}^{-1} \delta_{0} / \varepsilon_{0}$. If the radio cocoon has a boundary layer of thickness $d_{\mathrm{b}}=$ $5 \mathrm{kpc}$, which is isolating the interior from the exterior due to the lack of any magnetic flux exchange, then the escape frequency of these CRs is $v_{\text {layer }}=1.7 \mathrm{Gyr}^{-1} \delta_{0} / \varepsilon_{0}$. This is extremely slow for a low level of magnetic turbulence $\left(\delta_{0}<0.01\right)$.

If such a isolating layer does not exist, $\mathrm{CR}$ particles can reach the surface much easier and leave by cross field diffusion (over a short distance) or by following open field lines. Since only reconnection events of the fossil fields with the much weaker environmental fields could have opened the magnetic topology, $\eta_{\mathrm{s}} \ll 1$ is assumed due to the possible rarity of such events. Under such conditions the escape frequencies of $10 \mathrm{GeV}$ particles are $v_{\|} \approx 57 \mathrm{Gyr}^{-1} \eta_{\mathrm{s}} / \varepsilon_{0}$, and $v_{\perp} \approx 31 \mathrm{Gyr}^{-1} \delta_{0} / \varepsilon_{0}$. The CR streaming velocity along open flux tubes is $460 \mathrm{~km} \mathrm{~s}^{-1} / \varepsilon_{0}$ and therefore likely much below the cluster sound speed, so that CR self-confinement is not important for these particles. For a low level of magnetic turbulence $\left(\delta_{0}<0.01\right)$ and a mostly closed field topology $\left(\eta_{\mathrm{s}}<0.01\right)$ the escape of $10 \mathrm{GeV}$ particles requires several Gyr. If one considers further that such radio plasma is buoyant and therefore leaves the cluster centre within a few $100 \mathrm{Myr}$ (Churazov et al. 2001; Enßlin \& Heinz 2002), the fraction of CRs injected into a cluster core is expected to be small under such circumstances. This can impose constraints on several of the theoretical considerations listed in Sect. 1.1, which rely on an efficient escape of CR electrons, positrons or protons from radio cocoons into the centre of galaxy clusters.

Further, during the buoyant rise of a radio cocoon through a cluster atmosphere, the cocoon expands adiabatically. If all magnetic length-scales scale linearly with the cocoon size $L_{\mathrm{s}}$, and the magnetic field strength decreases adiabatically as $B \propto$ $L_{\mathrm{s}}^{-2}$ then all escape frequencies discussed here $\left(v_{\text {layer }}, v_{\|}, v_{\perp}\right)$ decrease according to $v \propto L_{\mathrm{s}}^{-2 / 3}$. This implies that CRs are better confined in a larger cocoon, or that CR escape is most rapidly at an early stage of the cocoon's buoyant voyage through the intra-cluster medium.

\subsection{Suddenly injected turbulence}

If a cluster merger event suddenly injects large-scale turbulence the radio plasma can become transparent even for low energy CR particles. The merger might produce turbulent flows with velocities of $v_{\mathrm{T}} \approx 1000 \mathrm{~km} \mathrm{~s}^{-1}$ on a scale of $l_{\mathrm{T}} \approx 100 \mathrm{kpc}$ which increase the turbulent magnetic energy density on large-scales by a factor of $X_{\mathrm{T}}=10$ from initially $\delta_{0}=0.01$ for example. For roughly an eddy turnover time $\tau_{\mathrm{T}}=l_{\mathrm{T}} / v_{\mathrm{T}} \approx 100 \mathrm{Myr}$ the small scale turbulence is not increased in regions far from shock waves. During only a tenth of this period (10 Myr, which is of the order the turbulent cascades needs to transfer energy from the scales of the radio cocoon $(10 \mathrm{kpc})$ down to the CR gyroradius length scales) an enhanced anomalous cross field escape should allow roughly $30 \% / \varepsilon_{0}$ of the $10 \mathrm{GeV}$ CR particles initially confined in the radio cocoon to escape. A similar number of the CRs would escape in the remaining $90 \mathrm{Myr}$ of fully developed turbulence. Significant losses would occur on even shorter timescales in the case that the pitch angle scattering efficiency is low $\left(\varepsilon_{0} \ll 1\right)$. The total loss of particles escaping along inter phase flux tubes is roughly $6 \%\left(\eta_{\mathrm{s}} / 0.01\right) / \varepsilon_{0} \ll 1$ during $0.1 \tau_{\mathrm{T}}$, and likely negligible if only a few $\mathrm{kpc}^{2}$ of the cocoons surface is opened $\left(\eta_{\mathrm{s}} \ll 0.01\right)$. If the cocoon would have an isolating boundary layer of thickness $5 \mathrm{kpc}$, then only $1.7 \%$ of the CR particles would be released during $0.1 \tau_{\mathrm{T}}$.

\subsection{Merger shock waves}

Cluster mergers happen frequently (Mohr et al. 1995; Jones \& Forman 1999; Schuecker et al. 2001) so that shock waves appear often in clusters (Quilis et al. 1998; Miniati et al. 2000), which should raise the turbulence level on all length scales during their passage. Therefore the strongly enhanced anomalous diffusivity discussed above is not expected to appear in this case. However, the turbulence level should increase substantially, leading to some enhancement of the particle escape rate, and the typical lengthscale $L_{\mathrm{s}}$ the particles have to travel before leaving the source also decreases substantially. Both effects in combination should lead to efficient particle escape.

The escape length $L_{\mathrm{s}}=V_{\mathrm{s}} / A_{\mathrm{s}}$ decreases due to two effects: first, the radio plasma volume shrinks by a factor $C=$ $V_{2} / V_{1}=\left(P_{1} / P_{2}\right)^{3 / 4}$ for radio plasma with a relativistic equation of state which is adiabatically (due to the high internal sound speed) compressed in an environmental shock wave with pressure jump $P_{2} / P_{1}$. Second, the shock wave should disrupt 
the radio plasma into filamentary or torus-like morphologies, as seen in numerical simulations (Enßlin \& Brüggen 2002) and in high resolution radio maps of some cluster radio relics (Slee et al. 2001). The numerical simulations show that a spherical radio cocoon transforms into a torus with major radius equal to the radius of the original sphere (the torus diameter is roughly that of the former sphere). Using the dimensions of the torus given by this observation and the above given volume shrinking factor $C$ one finds that the characteristic length-scale $L_{\mathrm{s}}=V_{\mathrm{s}} / A_{\mathrm{s}}$ decreases due to shock compression and change of the morphology by a factor

$$
\frac{L_{\mathrm{s}, 2}}{L_{\mathrm{s}, 1}}=\sqrt{\frac{3 C}{4 \pi}}\left[1+\sqrt{\frac{4 C}{3 \pi^{2}}}\right]^{-1} \approx \frac{1}{2} \sqrt{C} .
$$

Thus, for a typical merger shock wave with Mach number of the order 3 , and pressure jump roughly 10 the characteristic length scale decreases by a factor of nearly 6 . If at the same time the level of turbulence increases by an order of magnitude, the cross field diffusion escape rate is enlarged by nearly two orders of magnitude. Therefore also for radio plasma passing through a shock wave efficient CR particle escape is expected.

\section{Discussion}

A model has been presented describing the escape of CR particles from regions which are poorly magnetically connected to the environment. The main application of this theoryis to cocoons of radio galaxies.

In order to apply this model quantitatively to astrophysical systems, several poorly known parameters have to be determined, like the geometry of the source $\left(V_{\mathrm{s}}, A_{\mathrm{s}}, L_{\mathrm{s}}, \eta_{\mathrm{s}}, \sigma_{\mathrm{s}}\right)$, the field fluctuation length-scales $\left(\lambda_{\|}, \lambda_{\perp}, l_{\perp}, l_{B}\right)$, the nature and the level of the turbulence $\left(\delta_{0}\right)$, and the coupling strength between small-scale magnetic turbulence and the gyro-motion of charged particles $\left(\varepsilon_{0}\right)$. Several of these parameters are closely related and can roughly be estimated (e.g. the characteristic turbulence length-scales). Other parameters will remain unknown until a truly detailed knowledge of radio plasma is available.

However, even without detailed knowledge of these parameters, insight into the qualitative behaviour of CR transport between different phases is provided:

- An isolating magnetic layer at the surface of the CR confining region can efficiently suppress $\mathrm{CR}$ escape compared to the case in which flux tubes from the source interior touch (or even leave) the source surface.

- CR escape from a mostly magnetically confined region always requires parallel and cross field particle transport.

- The escape frequency of cosmic rays travelling along interphase flux tubes is independent of the level of turbulence (Eqs. (44)-(45)).

- The cross field escape frequency increases linearly with increasing turbulence level (Eqs. (56)-(58)).

- The parallel and perpendicular escape frequencies are mainly determined by the properties of the strong field region.

- All escape frequencies considered here have an identical scaling with the CR momentum, for any turbulence spectra.
E.g. for Kolmogorov turbulence it is $v_{\| / \perp} \propto p^{1 / 3}$, and for Kraichnan turbulence it is $v_{\| / \perp} \propto p^{1 / 2}$.

- In the case where the pitch angle scattering frequency is strongly reduced (Chandran 2000b) due to the possible anisotropic nature of weak magneto-hydrodynamic turbulence (Sridhar \& Goldreich 1994; Goldreich \& Sridhar 1997), this model can still be applicable as long as the particle transport is diffusive. In such a case one expects $\varepsilon_{0} \ll 1$.

- If the chaotic (topologically non-trivially twisted) part of the magnetic fluctuations extends down in the powerspectrum to the smallest length-scales, the anomalous cross field diffusion may be much larger than estimated here (Narayan \& Medvedev 2001) leading to a very rapid CR escape from radio cocoons. If this is indeed the case is not yet clear, but may be testable by observations of escaped particles 5 .

- In contrast to a statement by Chandran (2000a) it is shown here that the macroscopic diffusive transport of particles along a flux bundle with small scale inhomogeneities in the field strength is reduced by the presence of strong field bottlenecks (Appendix A).

- A sudden large-scale turbulence injection can lead temporarily to the enhanced anomalous diffusion regime. This increases both escape frequencies, but the cross field escape frequency by a larger factor.

- Cluster merger events can lead to strong particle losses from radio plasma. Shock waves disrupt the relativistic plasma into smaller pieces from which CRs can escape more easily. It should be noted that cluster merger waves and turbulence themself are able to accelerate CRs, which can mask (and/or energise) the CRs escaping from radio plasma.

- Merger can also lead to efficient particle escape from radio plasma not directly affected by shock waves. The enhanced anomalous diffusion due to the large-scale turbulence injected by the merger could also lead to efficient particle escape.

Given the large astrophysical importance of the problem of cosmic ray escape from radio plasma cocoons, further studies of this problem - theoretically and observationally - would be of great value. The presented work provides a starting point for such investigations.

Acknowledgements. I thank Benjamin Chandran, Eugene Churazov, Sebastian Heinz, Philipp P. Kronberg, and Kandu Subramanian for discussions and comments on the manuscript. This work was done in the framework of the EC Research and Training Network The Physics of the Intergalactic Medium.

\section{Appendix A: Homogenisation of the diffusion equation}

It is instructive to derive the macroscopic transport equation in the case that the spatial fluctuations in the parameters of Eq. (5)

5 E.g. annihilation of escaped positrons, decaying pions produced by relativistic protons, or even simply a low level diffuse synchrotron halo around a bright radio cocoon due to escaping electrons. 
can be described by a large-scale $x^{\prime}$ and a much smaller scale $\xi$, so that $x=x^{\prime}+\xi / \epsilon$ and $\epsilon$ is the ratio of the small to the largescale. A quantity $A(x)=A\left(x^{\prime}, \xi\right)$ is averaged over the smaller scale by $\langle A\rangle\left(x^{\prime}\right)=\lim _{\xi \rightarrow \infty} \int_{0}^{\xi} \mathrm{d} \xi^{\prime} A\left(x^{\prime}, \xi^{\prime}\right) / \xi$.

The evolution of the distribution function can be expressed as an asymptotic series in the small parameter $\epsilon$. To lowest order in $\epsilon$ this method of homogenisation gives

$$
\frac{\partial \bar{f}}{\partial t}+\frac{\partial}{\partial p}(\overline{\dot{p}} \bar{f})=\frac{\partial}{\partial x}\left(\bar{\kappa}_{\|} \frac{\partial(\bar{B} \bar{f})}{\bar{B} \partial x}\right)-\frac{\bar{f}}{\bar{\tau}}+\bar{q},
$$

where the prime was dropped $\left(x^{\prime} \rightarrow x\right)$. The details of this kind of calculation can be found in Holmes (1995). The homogenised parameters are given by

$$
\begin{aligned}
& \bar{f}=\langle f\rangle \\
& \bar{B}=\langle 1 / B\rangle^{-1} \\
& \overline{\dot{p}}=\bar{B}\langle\dot{p} / B\rangle \\
& \bar{\kappa}_{\|}=\bar{B} /\left\langle B / \kappa_{\|}\right\rangle \\
& \bar{\tau}=\bar{B}^{-1} /\langle 1 /(\tau B)\rangle \\
& \bar{q}=\langle q\rangle .
\end{aligned}
$$

In the case of vanishing small-scale fluctuations of all coefficients, their homogenised averages are, of course, unchanged by the homogenisation and Eq. (A.1) is identical to Eq. (5). In the case of small scale variations, the homogenised loss coefficients $\overline{\dot{p}}$ and $\bar{\tau}$ are weighted towards the weak field regions, where the flux tube has a larger diameter and therefore most of the particles reside. The effective large-scale diffusion coefficient $\bar{\kappa}_{\|}$is reduced compared to the typical small scale coefficient $\left\langle\kappa_{\|}\right\rangle$, due to the harmonic weighting in Eq. (A.5). The weight of these regions is further increased due to the expected anti-correlation of field strength and diffusion coefficient. However, even in the case that $\kappa_{\|}$does not change on the micro-scale, its global value is changed due to field strength fluctuations on the micro-scale: $\bar{\kappa}_{\|}=\kappa_{\|} /(\langle 1 / B\rangle\langle B\rangle) \leq \kappa_{\|}$ (equality only if $B(x, \xi)=B(x)$ ). If e.g. $10 \%$ of the micro-scale has a 100 times enhanced field strength, the averaged diffusion coefficient is a factor of 10 smaller than the microscopic one, in the case of a spatially constant $\kappa_{\|}$, otherwise much more. This demonstrates that magnetic traps modify the particle transport even in the diffusive regime, in contrast to an opposite statement by Chandran (2000a).

\section{Appendix B: Glossary}

$A_{\mathrm{s}}$ : source region surface area

CMB: Cosmic Microwave Background

CR: cosmic ray

$C$ : volume shrinking factor of a shock compressed radio cocoon

$\delta_{0}$ : relative strength of magnetic turbulence at reference scale (Eq. (13))

$D_{\mu \mu}$ : Fokker-Planck pitch angle diffusion coefficient

$d_{\mathrm{b}}$ : thickness of insulating boundary layer $d_{\mathrm{b}}=r_{\mathrm{s}}-r_{\mathrm{i}}$

$\varepsilon_{0}$ : fudge factor relating turbulence energy density to pitch angle scattering efficiency (Eq. (12))

$\varepsilon$ : ratio of scattering frequency to gyro-frequency (Eq. (12)) $\eta_{\mathrm{s}}$ : fraction of $A_{\mathrm{s}}$ occupied by inter-phase magnetic flux

$f$ : isotropic particle phase-space density per magnetic flux (Eq. (3))

$f_{\mathrm{s}}$ : average density of particles $(f)$ within the source

$\gamma$ : spectral index of magnetic turbulence spectrum

$g$ : isotropic particle phase space density per volume

$\kappa_{\|}$: parallel diffusion coefficient (Eqs. (6), (10) and (15))

$\kappa_{\perp}$ : perpendicular diffusion coefficient (Eqs. (11) and (16))

$\kappa_{\mathrm{a}}$ : anomalous cross field diffusion coefficient (Eqs. (19) and (20))

$\Lambda$ : Eqs. (17) and (18)

$\lambda_{\|}:$parallel correlation length of the field fluctuations

$\lambda_{\perp}$ : perpendicular correlation length of the field fluctuations, typical flux tube diameter

$l_{B}$ : magnetic turbulence reference scale (Eq. (13))

$l_{\perp}:\left\langle\boldsymbol{\delta} \boldsymbol{B}(\boldsymbol{x}) \cdot \boldsymbol{\delta} \boldsymbol{B}\left(\boldsymbol{x}+\boldsymbol{\xi}_{\perp}\right)\right\rangle=\delta B^{2}\left(l_{B}\right)\left(1-\frac{1}{2} \xi_{\perp}^{2} / l_{\perp}^{2}+O\left(\xi_{\perp}^{4}\right)\right)$ if only the chaotic (topologically non-trivial twisted) field fluctuations are regarded

$l_{\mathrm{S}}\left(l_{1}\right)$ : characteristic distance a CR travels before leaving a flux tube in the CR source (loss) region (Eq. (33))

$L_{\mathrm{s}}=V_{\mathrm{s}} / A_{\mathrm{s}}$ : characteristic length of source

$l_{\text {scatt }}:$ CR pitch-angle scattering length $l_{\text {scatt }}=v / v_{\mu}$

$M_{\|}:$number of inter phase flux tubes

$m$ : particle mass

$M_{\perp}$ : number of flux tubes forming the boundary

$\mu$ : cosine of the $\mathrm{CR}$ pitch angle

$N_{\mathrm{s}}$ : number of CR in source region

$v_{\|}$: CR escape frequency along flux tubes (Eqs. (40) and (44))

$v_{\mu}$ : particle-wave pitch-angle scattering frequency

$\nu_{\perp}$ : perpendicular escape frequency

$\phi_{B}$ : magnetic flux

$p$ : particle momentum

$q$ : isotropic particle phase-space injection rate per magnetic flux (Eq. (4))

$r_{\mathrm{g}}:$ particle gyro-radius

$r_{\mathrm{i}}$ : inner radius of (spherical) boundary layer

$r_{\mathrm{s}}$ : source radius in spherical approximation

$\sigma_{\perp}$ : fraction of flux tube surface in contact with loss region

$\tau$ : particle escape time from a flux tube

$\tau_{\mathrm{s}}$ : flux tube escape time in source region

$\tau_{\mathrm{T}}$ : eddy turn-over time

$v$ : particle velocity

$V_{\mathrm{s}}$ : source volume

$X_{\mathrm{T}}$ : increment factor of turbulence energy density

$y=\lambda_{\|} / l_{\mathrm{s}}$ : field correlation length over particle diffusion length (Eq. (54))

$Z$ : particle charge.

\section{References}

Bieber, J. W., \& Matthaeus, W. H. 1997, ApJ, 485, 655

Brunetti, G., Setti, G., Feretti, L., \& Giovannini, G. 2001, MNRAS, 320,365

Cesarsky, C. J. 1980, ARA\&A, 18, 289

Cesarsky, C. J., \& Völk, H. J. 1978, A\&A, 70, 367

Chandran, B. D. G. 2000a, ApJ, 529, 513

Chandran, B. D. G. 2000b, Phys. Rev. Lett., 85, 4656

Churazov, E., Brüggen, M., Kaiser, C. R., Böhringer, H., \& Forman, W. 2001, ApJ, 554, 261 
Dennison, B. 1980, ApJ, 239, L93

Duffy, P., Kirk, J. G., Gallant, Y. A., \& Dendy, R. O. 1995, A\&A, 302, L21

Enßlin, T. A., \& Brüggen, M. 2002, MNRAS, 331, 1011

Enßlin, T. A., \& Heinz, S. 2002, A\&A, 384, L27

Enßlin, T. A., \& Kaiser, C. R. 2000, A\&A, 360, 417

Enßlin, T. A., \& Sunyaev, R. A. 2002, A\&A, 383, 423

Felice, G. M., \& Kulsrud, R. M. 2001, ApJ, 553, 198

Furlanetto, S. R., \& Loeb, A. 2002, ApJ, 572, 796

Giacalone, J., \& Jokipii, J. R. 1999, ApJ, 520, 204

Giovannini, G., Feretti, L., Venturi, T., Kim, K. T., \& Kronberg, P. P. 1993, ApJ, 406, 399

Goldreich, P., \& Sridhar, S. 1997, ApJ, 485, 680

Hasselmann, K., \& Wibberenz, G. 1970, ApJ, 162, 1049

Heinz, S., \& Sunyaev, R. 2002, A\&A, 390, 751

Holman, G. D., Ionson, J. A., \& Scott, J. S. 1979, ApJ, 228, 576

Holmes, M. H. 1995, Introduction to Perturbation Methods (SpringerVerlag New York, Inc.)

Jokipii, J. R. 1966, ApJ, 146, 480

Jokipii, J. R. 1967, ApJ, 149, 405

Jones, C., \& Forman, W. 1999, ApJ, 511, 65

Klepach, E. G., \& Ptuskin, V. S. 1995, Astron. Lett., 21, 411

Kulsrud, R., \& Pearce, W. P. 1969, ApJ, 156, 445

Laing, R. A. 1980, MNRAS, 193, 439

Laing, R. A. 1981, ApJ, 248, 87

Michalek, G., \& Ostrowski, M. 1997, A\&A, 326, 793
Michalek, G., \& Ostrowski, M. 1998, A\&A, 337, 558

Michalek, G., Ostrowski, M., \& Schlickeiser, R. 1999, Sol. Phys., 184, 339

Miniati, F., Ryu, D., Kang, H., et al. 2000, ApJ, 542, 608

Mohr, J. J., Evrard, A. E., Fabricant, D. G., \& Geller, M. J. 1995, ApJ, 447,8

Narayan, R., \& Medvedev, M. V. 2001, ApJ, 562, L129

Quilis, V., Ibanez, J. M. A., \& Saez, D. 1998, ApJ, 502, 518

Rechester, A., \& Rosenbluth, M. 1978, Phys. Rev. Lett., 40, 38

Schlickeiser, R. 1989a, ApJ, 336, 243

Schlickeiser, R. 1989b, ApJ, 336, 264

Schlickeiser, R. 2002, Cosmic ray astrophysics (Springer, ISBN 3-540-66465-3)

Schuecker, P., Böhringer, H., Reiprich, T. H., \& Feretti, L. 2001, A\&A, 378, 408

Skilling, J. 1975a, Nature, 258, 687

Skilling, J. 1975b, MNRAS, 172, 557

Slee, O. B., Roy, A. L., Murgia, M., Andernach, H., \& Ehle, M. 2001, AJ, 122, 1172

Soker, N., Blanton, E. L., \& Sarazin, C. L. 2002, ApJ, 573, 533

Spangler, S. R., Myers, S. T., \& Pogge, J. J. 1984, AJ, 89, 1478

Sridhar, S., \& Goldreich, P. 1994, ApJ, 432, 612

Tademaru, E. 1969, ApJ, 158, 959

Wentzel, D. G. 1968, ApJ, 152, 987

Wentzel, D. G. 1969, ApJ, 156, 303 